\title{
Effect of a New Prokinetic Agent DA-9701 Formulated with Corydalis Tuber and Pharbitidis Semen on Cytochrome P450 and UDP-Glucuronosyltransferase Enzyme Activities in Human Liver Microsomes
}

\author{
Hye Young Ji, ${ }^{1}$ Kwang Hyeon Liu, ${ }^{2}$ Ji Hyeon Jeong, ${ }^{1}$ Dae-Young Lee, ${ }^{3}$ \\ Hyun Joo Shim, ${ }^{3}$ Miwon Son, ${ }^{3}$ and Hye Suk Lee ${ }^{1}$ \\ ${ }^{1}$ Drug Metabolism and Bioanalysis Laboratory, College of Pharmacy, The Catholic University of Korea, 43-1 Yeokgok 2-dong, \\ Wonmi-gu, Gyeonggi-do, Bucheon, 420-743, Republic of Korea \\ ${ }^{2}$ College of Pharmacy and Research Institute of Pharmaceutical Sciences, Kyungpook National University, \\ Daegu 702-701, Republic of Korea \\ ${ }^{3}$ Research Center, Dong-A Pharmaceutical Co., Yongin 446-905, Republic of Korea \\ Correspondence should be addressed to Hye Suk Lee, sianalee@catholic.ac.kr
}

Received 17 November 2011; Accepted 2 January 2012

Academic Editor: Alfredo Vannacci

Copyright () 2012 Hye Young Ji et al. This is an open access article distributed under the Creative Commons Attribution License, which permits unrestricted use, distribution, and reproduction in any medium, provided the original work is properly cited.

DA-9701 is a new botanical drug composed of the extracts of Corydalis tuber and Pharbitidis semen, and it is used as an oral therapy for the treatment of functional dyspepsia in Korea. The inhibitory potentials of DA-9701 and its component herbs, Corydalis tuber and Pharbitidis semen, on the activities of seven major human cytochrome P450 (CYP) enzymes and four UDPglucuronosyltransferase (UGT) enzymes in human liver microsomes were investigated using liquid chromatography-tandem mass spectrometry. DA-9701 and Corydalis tuber extract slightly inhibited UGT1A1-mediated etoposide glucuronidation, with $50 \%$ inhibitory concentration ( $\mathrm{IC}_{50}$ ) values of 188 and $290 \mu \mathrm{g} / \mathrm{mL}$, respectively. DA-9701 inhibited CYP2D6-catalyzed bufuralol $1^{\prime}$-hydroxylation with an inhibition constant $\left(K_{\mathrm{i}}\right)$ value of $6.3 \mu \mathrm{g} / \mathrm{mL}$ in a noncompetitive manner. Corydalis tuber extract competitively inhibited CYP2D6-mediated bufuralol 1'-hydroxylation, with a $K_{\mathrm{i}}$ value of $3.7 \mu \mathrm{g} / \mathrm{mL}$, whereas Pharbitidis semen extract showed no inhibition. The volume in which the dose could be diluted to generate an $\mathrm{IC}_{50}$ equivalent concentration (volume per dose index) value of DA-9701 for inhibition of CYP2D6 activity was 1.16 L/dose, indicating that DA-9701 may not be a potent CYP2D6 inhibitor. Further clinical studies are warranted to evaluate the in vivo extent of the observed in vitro interactions.

\section{Introduction}

Functional dyspepsia is a very common chronic gastrointestinal disorder, with a prevalence of $40 \%$ using the more liberal criteria [1]. Although the etiology and pathogenesis of functional dyspepsia are poorly understood, pathophysiologic abnormalities such as delayed gastric emptying and impaired gastric accommodation have been reported in $30-40 \%$ of functional dyspepsia patients [2-5]. Medications used for the treatment of functional dyspepsia have been developed based on the pathophysiological mechanisms associated with functional dyspepsia; those medications include prokinetic agents, which are now restricted due to serious adverse effects and failure to confirm their efficacy. Therefore, it is necessary to develop safer and more effective drugs for the treatment of functional dyspepsia.

DA-9701 is a new botanical drug composed of the extracts of Corydalis tuber from the roots of Corydalis yanhusuo W. T. Wang and Pharbitidis semen from the seed of Pharbitis nil Choisy, and it is used as an oral therapy (Motilitone ${ }^{\circledR}$ ) for the treatment of functional dyspepsia (FD) in Republic of Korea since May 2011 [6-8]. Pharbitidis semen has been used as a folk medicine for its analgesic effect on abdominal disorders. Corydalis tubers from the roots of Corydalis yanhusuo W. T. Wang have long been 
used as a herbal drug for their analgesic and antiulcer effects [9-11]. Corydaline and tetrahydroberberine, isoquinoline alkaloids of Corydalis tubers, promote gastric emptying and facilitate gastric accommodation [12, 13]. DA-9701 has been demonstrated to show strong gastroprokinetic effects and a safety profile superior to cisapride and mosapride $[7,8]$. The gastroprokinetic effects of DA-9701 might be mediated by the induction of pacemaker currents in the interstitial cells of Cajal (ICC) [6]. DA-9701 also has been shown to have antagonistic effects on the D2 receptor and agonistic effects on 5-HT4, 5-HT1a, and 5-HT1b receptors [7, 8].

Since botanical drugs share the same drug metabolizing enzymes, including cytochrome P450 (CYP) enzymes, UDP-glucuronosyltransferase (UGT) enzymes, and drug transporters, such as multidrug resistance protein and $\mathrm{p}$ glycoprotein, with commonly used drugs, the potential for herb-drug interaction is substantial [14-16]. Several medicinal herbs, including Dong quai (Angelica polymorpha), ginkgo (Ginkgo biloba), ginseng (Panax ginseng), milk thistle (Silybum marianum), licorice (Glycyrrhiza glabra), St. John's wort (Hypericum perforatum), and Woohwangcheongsimwon have been reported to cause herb-drug interactions [17-20]. Herb-drug interactions have been identified with St. John's wort that significantly involve reduced blood concentrations of cyclosporine, digoxin, midazolam, indinavir, tacrolimus, theophylline, and warfarin [21]. Undoubtedly, the early identification of herb-drug interactions is imperative to prevent potentially dangerous clinical outcomes.

To the best of our knowledge, no previous study has reported the effects of Corydalis tubers and Pharbitidis semen, the component herbs of DA-9701, on human CYP and UGT enzymes. In this study, the effects of DA-9701 and its component herbs, Corydalis tubers and Pharbitidis semen, on the activities of seven major human CYP enzymes and four major human UGTs, 1A1, 1A4, 1A9, and 2B7, were examined using pooled human liver microsomes in order to evaluate the possibility of drug interactions of DA-9701.

\section{Experimental}

2.1. Materials and Reagents. Corydaline, palmatine, and chlorogenic acid were obtained from Wako Pure Chemical Industries, Ltd. (Osaka, Japan). Acetaminophen, alamethicin, coumarin, diclofenac, etoposide, glucose-6-phosphate, glucose-6-phosphate dehydrogenase, 7-hydroxycoumarin, midazolam, $\beta$-nicotinamide adenine dinucleotide phosphate reduced form (NADPH), phenacetin, propofol, trifluoperazine, and uridine-5-diphosphoglucuronic acid trisodium salt (UDPGA) were purchased from Sigma Chemical Co. (St. Louis, MO, USA). Pooled human liver microsomes (H161), ${ }^{13} \mathrm{C}_{2},{ }^{15} \mathrm{~N}$-acetaminophen, bu-furalol, $N$-desethylamodiaquine, $1^{\prime}$-hydroxybufuralol, $\mathrm{d}_{9}-1^{\prime}$-hydroxybufuralol maleate, 4-hydroxydiclofenac, ${ }^{13} \mathrm{C}_{6}-4$ hydroxydiclofenac, 4-hydroxymephenytoin, $\mathrm{d}_{3}$-4-hydroxymephenytoin, 1' -hydroxymidazolam, and $(S)$ - mephenytoin were obtained from BD Gentest Co. (Woburn, MA, USA). Azidothymidine, azidothymidine glucuronide, and propofol glucuronide were obtained from Toronto Research Center
(Toronto, Canada). Acetonitrile and methanol (HPLC grade) were obtained from Burdick \& Jackson Inc. (Muskegon, MI, USA), and the other chemicals were of the highest quality available.

2.2. Preparation of DA-9701. DA-9701 is a standardized extract of Corydalis tuber and Pharbitidis semen which was prepared as previously reported [8]. Briefly, those dried herbs were mixed in a specific ratio $(5: 1)$ and extracted with $50 \%$ aqueous ethanol three times at room temperature for $48 \mathrm{~h}$. After filtration, the aqueous ethanol extract was evaporated under reduced pressure and lyophilized for a complete removal of the residual solvent to yield brown powder. Contents of two marker components, corydaline, an alkaloid, from Corydalis tuber and chlorogenic acid from Pharbitidis semen in DA-9701, were determined by high performance liquid chromatography (HPLC) [8].

Corydalis tuber extract and Pharbitidis semen extract were prepared as previously described [13]. Briefly, dried Corydalis tuber and Pharbitidis semen were separately extracted with $50 \%$ aqueous ethanol three times at room temperature for $48 \mathrm{~h}$. The solvents were removed under vacuum. The marker compounds including corydaline from Corydalis tuber extract and chlorogenic acid from Pharbitidis semen extract were also determined by HPLC [8]. The two raw materials were purchased in China.

\subsection{Inhibitory Effects of DA-9701 and Its Component} Herbs on Seven Major CYP Activities in Human Liver Microsomes. Inhibitory potencies ( $\mathrm{IC}_{50}$ values) of DA-9701, Corydalis tuber extract, and Pharbitidis semen extract were determined using CYP assays in the presence and absence of DA-9701, Corydalis tuber extract, and Pharbitidis semen extract using pooled human liver microsomes (H161, Gentest). Phenacetin O-deethylase, coumarin 7-hydroxylase, amodiaquine $N$-deethylase, diclofenac 4 -hydroxylase, $(S)$ mephenytoin 4-hydroxylase, bufuralol 1'-hydroxylase, and midazolam $1^{\prime}$-hydroxylase activities were determined as probe activities for CYP1A2, CYP2A6, CYP2C8, CYP2C9, CYP2C19, CYP2D6, and CYP3A, respectively, using cocktail incubation and liquid chromatography-tandem mass spectrometry (LC/MS/MS) [22]. The incubation mixtures were prepared in a total volume of $200 \mu \mathrm{L}$ as follows: pooled human liver microsomes $(0.25 \mathrm{mg} / \mathrm{mL}), 3.3 \mathrm{mM} \mathrm{MgCl}_{2}$, $50 \mathrm{mM}$ potassium phosphate buffer ( $\mathrm{pH} 7.4)$, and a cocktail of probe substrates and various concentrations of DA9701, Corydalis tuber extract, or Pharbitidis semen extract (final concentrations of $1-200 \mu \mathrm{g} / \mathrm{mL}$ with an acetonitrile concentration less than $0.5 \% \mathrm{v} / \mathrm{v})$. The substrates were used at concentrations approximately equal to their respective $K_{m}$ values: $50 \mu \mathrm{M}$ phenacetin, $2.5 \mu \mathrm{M}$ coumarin, $2.5 \mu \mathrm{M}$ amodiaquine, $10 \mu \mathrm{M}$ diclofenac, $100 \mu \mathrm{M}$ (S)mephenytoin, $5 \mu \mathrm{M}$ bufuralol, and $2.5 \mu \mathrm{M}$ midazolam. After a 3 -min preincubation period at $37^{\circ} \mathrm{C}$, the reactions were initiated by addition of an NADPH (final concentration of $1.3 \mathrm{mM}$ ) generating system and incubated for $20 \mathrm{~min}$ at $37^{\circ} \mathrm{C}$ in a shaking water bath. After incubation, the reaction was stopped by placement of the tubes on ice and 
addition of $100 \mu \mathrm{L}$ of ice-cold methanol containing internal standards $\left({ }^{13} \mathrm{C}_{2},{ }^{15} \mathrm{~N}\right.$-acetaminophen for acetaminophen and $N$-desethylamodiaquine, $\mathrm{d}_{5}-7$-hydroxycoumarin for 7-hydroxycoumarin, ${ }^{13} \mathrm{C}_{6}$-4-hydroxydiclofenac for 4hydroxydiclofenac, $\mathrm{d}_{3}$-4-hydroxy-mephenytoin for 4hydroxymephenytoin and 1'-hydroxymidazolam, $\mathrm{d}_{9}-1^{\prime}$ hydroxybufuralol for $1^{\prime}$-hydroxybufuralol). The incubation mixtures were then centrifuged at $13,000 \times \mathrm{g}$ for $5 \mathrm{~min}$. All incubations were performed in triplicate, and the mean values were used. For evaluation of NADPH-dependent mechanism-based inhibition of CYP activities, various concentrations of DA-9701, Corydalis tuber extract, or Pharbitidis semen extract $(1-200 \mu \mathrm{g} / \mathrm{mL})$ were preincubated for $30 \mathrm{~min}$ with human liver microsomes in the presence of NADPH. The reaction was started by addition of a cocktail of CYP probe substrates.

All seven metabolites produced from the cocktail incubation of CYP isoform-specific substrates were simultaneously determined by LC/MS/MS [22]. The system consisted of a tandem mass spectrometer (TSQ Quantum Access, ThermoFisher Scientific, San Jose, CA, USA) coupled with a Nanospace SI-2 LC system (Tokyo, Japan). Separation was performed on an Atlantis dC18 column $(5 \mu \mathrm{m}, 2.1 \mathrm{~mm}$ i.d. $\times 100 \mathrm{~mm}$, Waters, MA, USA) using the gradient elution of a mixture of $5 \%$ methanol in $0.1 \%$ formic acid (mobile phase A) and 95\% methanol in $0.1 \%$ formic acid (mobile phase B) at a flow rate of $0.25 \mathrm{~mL} / \mathrm{min}: 10 \%$ mobile phase $\mathrm{B}$ for $1 \mathrm{~min}, 10 \%$ to $95 \%$ mobile phase $\mathrm{B}$ for $1 \mathrm{~min}$, and $95 \%$ mobile phase B for $5 \mathrm{~min}$. The column and autosampler temperatures were 50 and $6^{\circ} \mathrm{C}$, respectively. After $1.5 \mathrm{~min}$, the LC eluent was diverted from waste to the mass spectrometer fitted with the electrospray ionization (ESI) source and operated in positive ion mode. ESI source settings for ionization of the metabolites were as follows: electrospray voltage, $5.0 \mathrm{kV}$; vaporizer temperature, $420^{\circ} \mathrm{C}$; capillary temperature $360^{\circ} \mathrm{C}$; sheath gas pressure, $35 \mathrm{psi}$; auxiliary gas pressure, $10 \mathrm{psi}$. Quantification was performed by selected reaction monitoring (SRM) of the $[\mathrm{M}+\mathrm{H}]^{+}$ ion and the related product ion for each metabolite. SRM transitions for the metabolites and internal standards are summarized in our previous paper [22]. Analytical data were processed using Xcalibur software (Thermo Fisher Scientific).

\subsection{Inhibitory Effects of DA-9701 and Its Component} Herbs on Four UGT Activities in Human Liver Microsomes. The inhibitory potencies ( $\mathrm{IC}_{50}$ values) of DA-9701, Corydalis tuber extract, or Pharbitidis semen extract were also determined with UGT assays in the presence and absence of DA-9701, Corydalis tuber extract, or Pharbitidis semen extract using pooled human liver microsomes. Etoposide glucuronidation, trifluoperazine glucuronidation, propofol glucuronidation, and azidothymidine glucuronidation activities were determined as probe activities for UGT1A1, UGT1A4, UGT1A9, and UGT2B7, respectively, using LC/MS/MS [23]. Incubation mixtures were prepared in a total volume of $100 \mu \mathrm{L}$ as follows: pooled human liver microsomes $(0.2 \mathrm{mg} / \mathrm{mL}$ for etoposide, trifluoperazine, and azidothymidine; $0.1 \mathrm{mg} / \mathrm{mL}$ for propofol), $25 \mu \mathrm{g} / \mathrm{mL}$ alamethicin, $10 \mathrm{mM} \mathrm{MgCl}_{2}, 50 \mathrm{mM}$ tris buffer ( $\mathrm{pH}$ 7.4), each UGT-isoform specific probe substrate $(200 \mu \mathrm{M}$ etoposide for UGT1A1, $5 \mu \mathrm{M}$ trifluoperazine for UGT1A4, $10 \mu \mathrm{M}$ propofol for UGT1A9, or $100 \mu \mathrm{M}$ azidothymidine for UGT2B7), and various concentrations of DA-9701, Corydalis tuber extract, or Pharbitidis semen extract (final concentrations of $1-200 \mu \mathrm{g} / \mathrm{mL}$ with acetonitrile concentration less than $0.5 \% \mathrm{v} / \mathrm{v})$. Reactions were initiated by addition of UDPGA (final concentration of $5 \mathrm{mM}$ ), and incubations were carried out at $37^{\circ} \mathrm{C}$ in a shaking water bath for $30 \mathrm{~min}$. Reactions were terminated by addition of $100 \mu \mathrm{L}$ of ice-cold methanol containing an internal standard $(100 \mathrm{ng} / \mathrm{mL}$ ezetimibe for etoposide glucuronide and propofol glucuronide; $30 \mathrm{ng} / \mathrm{mL}$ meloxicam for trifluoperazine glucuronide and azidothymidine glucuronide). The incubation mixtures were centrifuged at $13,000 \times \mathrm{g}$ for $5 \mathrm{~min}$, followed by dilution of $40 \mu \mathrm{L}$ of the supernatant with $60 \mu \mathrm{L}$ of water. The aliquot $(5 \mu \mathrm{L})$ was injected onto LC/MS/MS. All incubations were performed in duplicate and the mean values were used.

Glucuronides produced from UGT isoform-specific substrates were, respectively, determined by LC/MS/MS [23]. Separation was performed on an Atlantis dC18 column $(5 \mu \mathrm{m}, 2.1 \mathrm{~mm}$ i.d. $\times 100 \mathrm{~mm}$, Waters, MA, USA) using the gradient elution of a mixture of $5 \%$ methanol in $0.1 \%$ formic acid (mobile phase A) and $95 \%$ methanol in $0.1 \%$ formic acid (mobile phase B) at a flow rate of $0.25 \mathrm{~mL} / \mathrm{min}: 10 \%$ mobile phase B for $2 \mathrm{~min}, 10 \%$ to $95 \%$ mobile phase B for $4 \mathrm{~min}$. The column and autosampler temperatures were 50 and $6^{\circ} \mathrm{C}$, respectively. After $3.0 \mathrm{~min}$, the LC eluent was diverted from waste to the mass spectrometer fitted with an ESI source. ESI source settings for ionization of trifluoperazine glucuronide and azidothymidine glucuronide in positive ion mode were as follows: electrospray voltage, $5.0 \mathrm{kV}$; vaporizer temperature, $420^{\circ} \mathrm{C}$; capillary temperature $360^{\circ} \mathrm{C}$; sheath gas pressure, $35 \mathrm{psi}$; auxiliary gas pressure, $10 \mathrm{psi}$. ESI source settings for ionization of etoposide glucuronide and propofol glucuronide in negative ion mode were as follows: electrospray voltage, $-4.0 \mathrm{kV}$; vaporizer temperature, $420^{\circ} \mathrm{C}$; capillary temperature $360^{\circ} \mathrm{C}$; sheath gas pressure, $35 \mathrm{psi}$; auxiliary gas pressure, $10 \mathrm{psi}$. Quantification was performed by SRM of the $[\mathrm{M}+\mathrm{H}]^{+}$ion for trifluoperazine glucuronide and azidothymidine glucuronide or $[\mathrm{M}-\mathrm{H}]^{-}$for etoposide glucuronide and propofol glucuronide and the related product ion for each metabolite. SRM transitions for the metabolites and internal standard are summarized in our previous paper [23]. Analytical data were processed using Xcalibur software (Thermo Fisher Scientific).

2.5. Kinetic Analysis. In order to determine $K_{\mathrm{i}}$ values of DA9701, Corydalis tuber extract, and a typical inhibitor quinidine for CYP2D6 enzyme, human liver microsomes $(0.1 \mathrm{mg} /$ $\mathrm{mL}$ ) were incubated with various concentrations of 0.5 $5 \mu \mathrm{M}$ bufuralol, $10 \mathrm{mM} \mathrm{MgCl}_{2}$, and various concentrations of DA-9701, Corydalis tuber extract, or quinidine in $50 \mathrm{mM}$ potassium phosphate buffer ( $\mathrm{pH}$ 7.4) in a total incubation volume of $200 \mu \mathrm{L}$. Reactions were initiated by addition of $\mathrm{NADPH}$ (final concentration of $1 \mathrm{mM}$ ) at $37^{\circ} \mathrm{C}$ and stopped 
TABle 1: Effects of DA-9701, Corydalis tuber extract (CT Ex), and Pharbitidis semen extract (PS Ex) on CYP metabolic activity in pooled human liver microsomes, H161 using cocktail substrate assay.

\begin{tabular}{|c|c|c|c|c|c|c|c|}
\hline \multirow{3}{*}{ CYP activity } & \multirow{3}{*}{ CYP } & \multicolumn{6}{|c|}{$\mathrm{IC}_{50}, \mu \mathrm{g} / \mathrm{mL}\left(\mathrm{VDI}^{* *}, \mathrm{~L} /\right.$ dose $)$} \\
\hline & & \multicolumn{3}{|c|}{ No preincubation } & \multicolumn{3}{|c|}{ With preincubation* } \\
\hline & & DA-9701 & CT Ex & PS Ex & DA-9701 & CT Ex & PS Ex \\
\hline Phenacetin $O$-deethylation & $1 \mathrm{~A} 2$ & N.I. ${ }^{* * *}$ & N.I. & N.I. & N.I. & N.I. & N.I. \\
\hline Coumarin 7-hydroxylation & $2 \mathrm{~A} 6$ & N.I. & N.I. & N.I. & N.I. & N.I. & N.I. \\
\hline Amodiaquine $N$-deethylation & $2 \mathrm{C} 8$ & N.I. & N.I. & N.I. & N.I. & N.I. & N.I. \\
\hline Diclofenac 4-hydroxylation & $2 \mathrm{C} 9$ & N.I. & N.I. & N.I. & N.I. & N.I. & N.I. \\
\hline S-mephenytoin $4^{\prime}$-hydroxylation & $2 \mathrm{C} 19$ & N.I. & 145.9 & N.I. & $167.6(0.18)^{* *}$ & 130.3 & N.I. \\
\hline Bufuralol 1'-hydroxylation & 2D6 & $25.9(1.16)^{* *}$ & 15.8 & N.I. & $34.3(0.88)^{* *}$ & 16.7 & N.I. \\
\hline Midazolam 1'-hydroxylation & $3 \mathrm{~A} 4$ & N.I. & N.I. & N.I. & N.I. & N.I. & N.I. \\
\hline
\end{tabular}

* DA-9701, Corydalis tuber extract (CT Ex), and Pharbitidis semen extract (PS Ex) were preincubated for 30 min in the presence of NADPH before the addition of the substrate. ${ }^{* *}$ VDI: volume per dose index, $* * *$ N.I.: no inhibition at $200 \mu \mathrm{g} / \mathrm{mL}$ of DA-9701. Cocktail substrate concentrations used for the assessment of $\mathrm{IC}_{50}$ were as follows: $50 \mu \mathrm{M}$ phenacetin, $2.5 \mu \mathrm{M}$ coumarin, $2.5 \mu \mathrm{M}$ amodiaquine, $10 \mu \mathrm{M}$ diclofenac, $100 \mu \mathrm{M}(S)$-mephenytoin, $5.0 \mu \mathrm{M}$ bufuralol, and $2.5 \mu \mathrm{M}$ midazolam. The data represent the average of three determinations.

TABLE 2: $K_{\mathrm{i}}$ values for the inhibition of CYP2D6-catalyzed bufuralol $1^{\prime}$-hydroxylation activity by DA-9701, Corydalis tuber extract, and quinidine in pooled human liver microsomes, H161.

\begin{tabular}{lcc}
\hline Substances & $K_{\mathrm{i}}$ & Inhibition mode \\
\hline DA-9701 & $6.3 \mu \mathrm{g} / \mathrm{mL}$ & Noncompetitive \\
Corydalis tuber extract & $3.7 \mu \mathrm{g} / \mathrm{mL}$ & Competitive \\
Quinidine & $0.038 \mu \mathrm{M}$ & Noncompetitive \\
\hline
\end{tabular}

after $15 \mathrm{~min}$ by placement of the incubation tubes on ice and addition of $100 \mu \mathrm{L}$ of ice-cold methanol containing an internal standard $\left(0.5 \mu \mathrm{g} / \mathrm{mL} \mathrm{d}_{9}-1^{\prime}\right.$-hydroxybufuralol). The incubation mixtures were centrifuged at $13,000 \times \mathrm{g}$ for $5 \mathrm{~min}$, and aliquots $(5 \mu \mathrm{L})$ of the supernatants were analyzed by LC/MS/MS.

2.6. Data Analysis. $\mathrm{IC}_{50}$ values (concentration of inhibitor causing 50\% inhibition of the original enzyme activity) were calculated using WinNonlin software, a nonlinear regression analysis program (Pharsight, Mountain View, CA, USA). Apparent kinetic parameters for inhibitory potential $\left(K_{\mathrm{i}}\right.$ values) were estimated from the fitted curves using Enzyme Kinetics Ver. 1.3 program (Systat Software Inc., San Jose, CA, USA).

\section{Results and Discussion}

The inhibitory effects of DA-9701, Corydalis tuber extract, and Pharbitidis semen extract on seven major human CYP isoforms were evaluated using cocktail CYP probe substrates in human liver microsomes. CYP2D6-catalyzed bufuralol 1'-hydroxylation activity was inhibited by DA9701 and Corydalis tuber extract with $\mathrm{IC}_{50}$ values of 25.9 and $15.8 \mu \mathrm{g} / \mathrm{mL}$, respectively, but not by Pharbitidis semen extract (Table 1). DA-9701, Corydalis tuber extract, and Pharbitidis semen extract at $200 \mu \mathrm{g} / \mathrm{mL}$ showed negligible inhibition on CYP1A2-mediated phenacetin $O$ deethylation, CYP2A6-mediated coumarin 7-hydroxylation, CYP2C8-mediated amodiaquine $N$-deethylation, CYP2C9mediated diclofenac 4-hydroxylation, CYP2C19-mediated $[S]$-mephenytoin 4'-hydroxylation, and CYP3A-mediated midazolam 1 '-hydroxylation. $\mathrm{IC}_{50}$ values of corydaline and palmatine, the marker compounds of DA-9701 for CYP2D6catalyzed bufuralol 1' -hydroxylation activity were 23.8 [23] and $49.3 \mu \mathrm{g} / \mathrm{mL}$ (our unpublished data), respectively.

Although corydaline and palmatine inhibited CYP2D6 isozyme, they are not the major constituents causing the inhibition of CYP2D6 activity, because their contents in DA9701 (corydaline, $0.85 \mu \mathrm{g} / \mathrm{mg}$ and palmatine, $0.13 \mu \mathrm{g} / \mathrm{mg} \mathrm{DA}-$ 9701) and Corydalis tuber extract (corydaline, $1.12 \mu \mathrm{g} / \mathrm{mg}$ and palmatine, $0.18 \mu \mathrm{g} / \mathrm{mg}$ Corydalis tuber extract) are very low. Therefore, other constituents may contribute to the inhibition of CYP2D6 activity by DA-9701 and Corydalis tuber extract. Further studies are needed to explore how the constituents of those compounds contribute to the inhibition of human CYP2D6 isozyme. The inhibitory potencies of DA-9701, Corydalis tuber extract, and Pharbitidis semen extract were not significantly affected after a 30-min period of preincubation with human liver microsomes in the presence of NADPH (Table 1), suggesting that a mechanismbased inhibitory component was not present in DA-9701, Corydalis tuber extract, and Pharbitidis semen.

Volume per dose index (VDI) is defined as the volume in which one dose would be diluted to obtain the corresponding $\mathrm{IC}_{50}$ concentration, as described by Strandell et al. [24], to determine the potential for in vivo inhibition of herbal preparations. If VDI value for a herbal preparation approaches $4 \mathrm{~L}$, corresponding to human blood volume, the potential enzyme interaction of herbal preparation with pharmaceuticals should be further investigated [24]. Since a recommended human dose for DA-9701 is $30 \mathrm{mg}$, VDI value of DA-9701 for inhibition of CYP2D6 activity was $1.16 \mathrm{~L} /$ dose, suggesting that DA-9701 may not be a potent CYP2D6 inhibitor. 


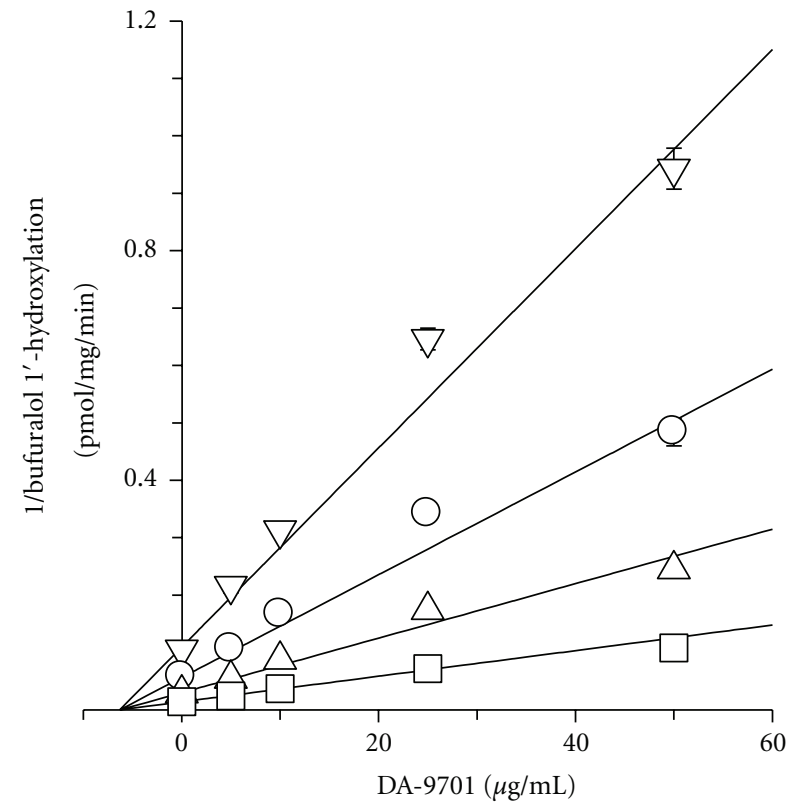

(a)

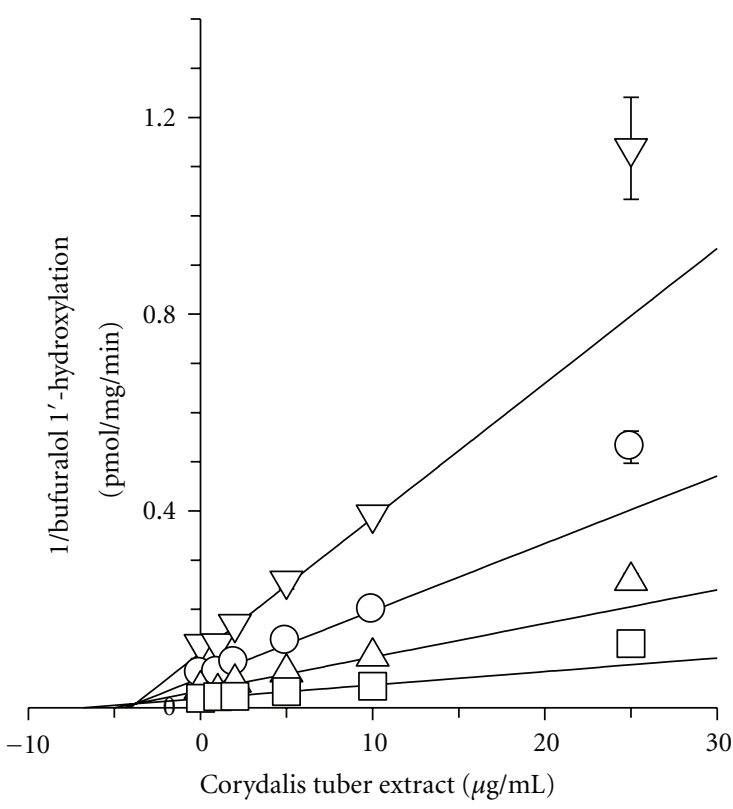

(b)

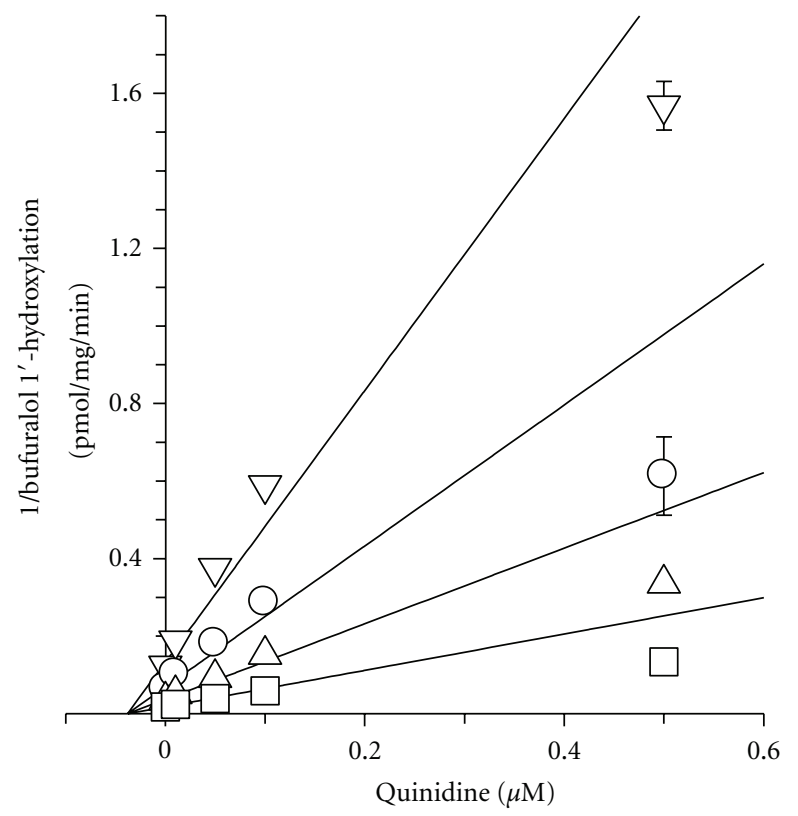

(c)

FIGURE 1: Representative Dixon plots for inhibitory effects of (a) DA-9701, (b) Corydalis Tuber extract, and (c) quinidine on CYP2D6catalyzed bufuralol 1'-hydroxylation in pooled human liver microsomes, H161. Each symbol represents the bufuralol concentration: $0.5 \mu \mathrm{M}$ $(\nabla), 1.0 \mu \mathrm{M}(\bigcirc), 2.0 \mu \mathrm{M}(\triangle)$, and $5.0 \mu \mathrm{M}(\square)$. Each data point represents the mean of triplicate experiments.

TABle 3: Effects of DA-9701, Corydalis tuber extract, and Pharbitidis semen extract on UGT metabolic activity in pooled human liver microsomes, H161.

\begin{tabular}{lcccc}
\hline UGT & Marker enzyme & \multicolumn{1}{c}{$\mathrm{IC}_{50}(\mu \mathrm{g} / \mathrm{mL})$} & Pharbitidis semen extract \\
\hline UGT1A1 & Etoposide glucuronidation & 188 & Corydalis tuber extract & No inhibition* \\
UGT1A4 & Trifluoperazine N-glucuronidation & No inhibition & No inhibition & No inhibition \\
UGT1A9 & Propofol glucuronidation & No inhibition & No inhibition & No inhibition \\
UGT2B7 & Azidothymidine glucuronidation & No inhibition & No inhibition & No inhibition \\
\hline
\end{tabular}

${ }^{*}$ There was no inhibition at $200 \mu \mathrm{g} / \mathrm{mL}$ of DA-9701, Corydalis tuber extract, and Pharbitidis semen extract. The data represent the average of duplicate analysis. 
In an inhibition study, the apparent $K_{\mathrm{i}}$ value is a better parameter for defining the interaction of the inhibitor with a particular enzyme. $K_{\mathrm{i}}$ values and inhibition types (competitive, noncompetitive, uncompetitive, or mixed) for DA9701 and Corydalis tuber extract were initially estimated by graphical methods such as Lineweaver-Burk plot and Dixon plot, but they were ultimately determined by nonlinear leastsquare regression analysis for the best enzyme inhibition model using Enzyme kinetics software. DA-9701 showed noncompetitive inhibition for CYP2D6-catalyzed bufuralol $1^{\prime}$-hydroxylation, with a $K_{i}$ value of $6.3 \mu \mathrm{g} / \mathrm{mL}$ (Table 2, Figure 1). Corydalis tuber extract competitively inhibited CYP2D6-catalyzed bufuralol 1'-hydroxylation, with a $K_{\mathrm{i}}$ value of $3.7 \mu \mathrm{g} / \mathrm{mL}$ (Table 2, Figure 1). The inhibitory potential of corydaline on CYP2D6 activity $\left(K_{\mathrm{i}}\right.$ value of $10.1 \mu \mathrm{g} / \mathrm{mL}$ $(27.3 \mu \mathrm{M}))$ [23] was not found to be strong, as compared with the inhibition produced by quinidine $\left[K_{\mathrm{i}}\right.$ value of $12 \mathrm{ng} / \mathrm{mL}(0.038 \mu \mathrm{M})]$, a selective inhibitor of CYP2D6 $[25,26]$.

We also evaluated the inhibitory potential of DA-9701, Corydalis tuber extract, and Pharbitidis semen extract on four major UGT isoform activities. DA-9701 and Corydalis tuber extract inhibited UGT1A1-mediated etoposide glucuronidation, with $\mathrm{IC}_{50}$ values of 188 and $290 \mu \mathrm{g} /$ $\mathrm{mL}$, respectively. UGT1A4-mediated trifluoperazine $N$ glucuronidation, UGT1A9-mediated propofol glucuronidation, and UGT2B7-mediated azidothymidine glucuronidation were not inhibited by treatment with DA-9701 and Corydalis tuber extract (Table 3 ). Pharbitidis semen extract showed no inhibition of UGT1A1, UGT1A4, UGT1A9, and UGT2B7 activities in human liver microsomes (Table 3 ). VDI value of DA-9701 for inhibition of UGT1A1 activity was $0.16 \mathrm{~L} /$ dose, suggesting that DA-9701 may not inhibit in vivo UGT1A1 activity. This was supported by the comparison with $\mathrm{IC}_{50}$ values of UGT1A1 inhibitors, such as ritonavir $\left(\mathrm{IC}_{50}=1.7 \mu \mathrm{M}\right)$ and ketoconazole $\left(\mathrm{IC}_{50}=7.3 \mu \mathrm{M}\right)$ [27].

CYP2D6 catalyzes oxidation of a wide range of substrates, including desipramine, dextromethorphan, haloperidol, $S$-metoprolol, nortryptyline, paroxetine, and tamoxifen (http://medicine.iupui.edu/clinpharm/ddis/). Compared to quinidine, a known inhibitor of CYP2D6-catalyzed bufuralol $1^{\prime}$-hydroxylation $[25,26,28]$, DA-9701 is a weak inhibitor $\left(K_{\mathrm{i}}, 12 \mathrm{ng} / \mathrm{mL}\right.$ versus $\left.6.3 \mu \mathrm{g} / \mathrm{mL}\right)$. On the basis of an $\mathrm{IC}_{50}$ value and VDI value of $1.16 \mathrm{~L}$ for a $30 \mathrm{mg}$ dose, DA-9701 will be expected to have weak, if any, inhibition of CYP2D6catalyzed metabolism in vivo. However, no pharmacokinetic data on DA-9701 are available. False prediction of in vivo drug-drug interactions from in vitro data may be occurred as a result of mechanism-based inhibition, plasma protein binding of inhibitors, production of inhibitory metabolites, and/or hepatic uptake. Therefore, the results need to be confirmed in clinical studies.

\section{Conclusions}

In conclusion, the effects of DA-9701 and its component herbs, Corydalis tuber extract and Pharbitidis semen extract, on seven CYPs and four UGTs were evaluated across a wide range of substrates using human liver microsomes in vitro. Pharbitidis semen extract showed no inhibition of seven CYPs and four UGTs in human liver microsomes. CYP2D6 activity was moderately inhibited by DA-9701 and Corydalis tuber extract during incubation with NADPH in human liver microsomes. On the basis of a $K_{i}$ value and VDI value, DA-9701 will be expected to have weak, if any, inhibition of CYP2D6-catalyzed metabolism in vivo. Those results suggest that high uptake of botanical drug DA-9701 or Corydalis tuber extract may cause an interaction with drugs metabolized by CYP2D6 in some individuals. It is important to note, however, that the inhibition of CYP activities in vitro does not necessarily translate into drug interactions in clinical situations. Clinical trials to evaluate the inhibitory effects of DA-9701 on CYP2D6 remain to be conducted.

\section{Conflict of Interests}

The authors declare no conflict of interests.

\section{Authors' Contributions}

H. Ji and K. Liu contributed equally to this work.

\section{Acknowledgments}

This work was supported by the National Research Foundation of Korea (NRF) Grant funded by the Ministry of Education, Science and Technology (2011-0018631) and the Global Leading Technology Program of the Office of Strategic R\&D Planning (OSP), funded by the Ministry of Knowledge Economy, Republic of Korea (10039303).

\section{References}

[1] H. B. El-Serag and N. J. Talley, "Systematic review: the prevalence and clinical course of functional dyspepsia," Alimentary Pharmacology and Therapeutics, vol. 19, no. 6, pp. 643-654, 2004.

[2] G. E. Boeckxstaens, D. P. Hirsch, S. D. Kuiken, S. H. Heisterkamp, and G. N. J. Tytgat, "The proximal stomach and postprandial symptoms in functional dyspeptics," The American Journal of Gastroenterology, vol. 97, no. 1, pp. 40-48, 2002.

[3] B. D. Maes, Y. F. Ghoos, M. I. Hiele, and P. J. Rutgeerts, "Gastric emptying rate of solids in patients with nonulcer dyspepsia," Digestive Diseases and Sciences, vol. 42, no. 6, pp. 1158-1162, 1997.

[4] G. Sarnelli, P. Caenepeel, B. Geypens, J. Janssens, and J. Tack, "Symptoms associated with impaired gastric emptying of solids and liquids in functional dyspepsia," The American Journal of Gastroenterology, vol. 98, no. 4, pp. 783-788, 2003.

[5] B. D. J. van den Elzen and G. E. E. Boeckxstaens, "Review article: a critical view on impaired accommodation as therapeutic target for functional dyspepsia," Alimentary Pharmacology and Therapeutics, vol. 23, no. 11, pp. 1499-1510, 2006.

[6] S. Choi, J. J. Choi, J. Y. Jun et al., "Induction of pacemaker currents by DA-9701, a prokinetic agent, in interstitial cells of Cajal from murine small intestine," Molecules and Cells, vol. 27, no. 3, pp. 307-312, 2009. 
[7] E. R. Kim, B. H. Min, S. O. Lee, T. H. Lee, M. Son, and P. L. Rhee, "The effects of DA-9701, a novel prokinetic agent, on gastric accommodation in conscious dogs," Journal of Gastroenterology and Hepatology. In press.

[8] T. H. Lee, J. J. Choi, D. H. Kim et al., "Gastroprokinetic effects of DA-9701, a new prokinetic agent formulated with Pharbitis Semen and Corydalis Tuber," Phytomedicine, vol. 15, no. 10, pp. 836-843, 2008.

[9] M. Kubo, H. Matsuda, K. Tokuoka, S. Ma, and H. Shiomoto, "Anti-inflammatory activities of methanolic extract and alkaloidal components from Corydalis tuber," Biological and Pharmaceutical Bulletin, vol. 17, no. 2, pp. 262-265, 1994.

[10] H. Matsuda, K. Tokuoka, J. Wu, T. Tanaka, and M. Kubo, "Inhibitory effects of methanolic extract from corydalis tuber against types I-IV allergic models," Biological and Pharmaceutical Bulletin, vol. 18, no. 7, pp. 963-967, 1995.

[11] C. S. Yuan, S. R. Mehendale, C. Z. Wang et al., "Effects of Corydalis yanhusuo and Angelicae dahuricae on cold pressorinduced pain in humans: a controlled trial," Journal of Clinical Pharmacology, vol. 44, no. 11, pp. 1323-1327, 2004.

[12] T. H. Lee, K. H. Kim, S. O. Lee, K. R. Lee, M. Son, and M. Jin, "Tetrahydroberberine, an isoquinoline alkaloid isolated from corydalis tuber, enhances gastrointestinal motor function," Journal of Pharmacology and Experimental Therapeutics, vol. 338, no. 3, pp. 917-924, 2011.

[13] T. H. Lee, M. Son, and S. Y. Kim, "Effects of corydaline from corydalis tuber on gastric motor function in an animal model," Biological and Pharmaceutical Bulletin, vol. 33, no. 6, pp. 958962, 2010.

[14] M.-E. F. Mohamed and R. F. Frye, "Effects of herbal supplements on drug glucuronidationreview of clinical, animal, and in vitro studies," Planta Medica, vol. 77, no. 4, pp. 311-321, 2011.

[15] D. H. Na, H. Y. Ji, E. J. Park, M. S. Kim, K.-H. Liu, and H. S. Lee, "Evaluation of metabolism-mediated herb-drug interactions," Archives of Pharmacal Research, vol. 34, no. 11, pp. 1829-1842, 2011.

[16] S. F. Zhou, Z. W. Zhou, C. G. Li et al., "Identification of drugs that interact with herbs in drug development," Drug Discovery Today, vol. 12, no. 15-16, pp. 664-673, 2007.

[17] S.-M. He, E. Chan, and S.-F. Zhou, "ADME properties of herbal medicines in humans: evidence, challenges andstrategies," Current Pharmaceutical Design, vol. 17, no. 4, pp. 357407, 2011.

[18] S. M. He, A. K. Yang, X. T. Li, Y. M. Du, and S. F. Zhou, "Effects of herbal products on the metabolism and transport of anticancer agents," Expert Opinion on Drug Metabolism and Toxicology, vol. 6, no. 10, pp. 1195-1213, 2010.

[19] H. Kim, K. B. Kim, H. Y. Ku et al., "Identification and characterization of potent CYP2B6 inhibitors in Woohwangcheongsimwon suspension, an herbal preparation used in the treatment and prevention of apoplexy in Korea and China," Drug Metabolism and Disposition, vol. 36, no. 6, pp. 1010-1015, 2008.

[20] D. K. Sevior, J. Hokkanen, A. Tolonen et al., "Rapid screening of commercially available herbal products for the inhibition of major human hepatic cytochrome P450 enzymes using the Nin-one cocktail,” Xenobiotica, vol. 40, no. 4, pp. 245-254, 2010.

[21] X.-W. Chen, E. S. Serag, K. B. Sneed et al., "Clinical herbal interactions with conventional drugs: from molecules to maladies," Current Medicinal Chemistry, vol. 18, no. 31, pp. 4836-4850, 2011.

[22] H. Y. Ji, S. Y. Kim, D. K. Kim, J. H. Jeong, and H. S. Lee, "Effects of eupatilin and jaceosidin on cytochrome P450 enzyme activities in human liver microsomes," Molecules, vol. 15, no. 9, pp. 6466-6474, 2010.

[23] H. Y. Ji, K. H. Liu, H. Lee et al., "Corydaline inhibits multiple cytochrome P450 and UDP-glucuronosyltransferase enzyme activities in human liver microsomes," Molecules, vol. 16, no. 8, pp. 6591-6602, 2011.

[24] J. Strandell, A. Neil, and G. Carlin, "An approach to the in vitro evaluation of potential for cytochrome P450 enzyme inhibition from herbals and other natural remedies," Phytomedicine, vol. 11, no. 2-3, pp. 98-104, 2004.

[25] E. A. Dierks, K. R. Stams, H. K. Lim, G. Cornelius, H. Zhang, and S. E. Ball, "A method for the simultaneous evaluation of the activities of seven major human drugmetabolizing cytochrome P450s using an in vitro cocktail of probe substrates and fast gradient liquid chromatography tandem mass spectrometry," Drug Metabolism and Disposition, vol. 29, no. 1, pp. 23-29, 2001.

[26] M. J. Kim, H. Kim, I. J. Cha et al., "High-throughput screening of inhibitory potential of nine cytochrome P450 enzymes in vitro using liquid chromatography/tandem mass spectrometry," Rapid Communications in Mass Spectrometry, vol. 19, no. 18, pp. 2651-2658, 2005.

[27] J. Zhou, T. S. Tracy, and R. P. Remmel, "Correlation between bilirubin glucuronidation and estradiol-3-gluronidation in the presence of model UDP-glucuronosyltransferase 1A1 substrates/inhibitors," Drug Metabolism and Disposition, vol. 39, no. 2, pp. 322-329, 2011.

[28] D. J. Newton, R. W. Wang, and A. Y. H. Lu, "Cytochrome P450 inhibitors: evaluation of specificities in the in vitro metabolism of therapeutic agents by human liver microsomes," Drug Metabolism and Disposition, vol. 23, no. 1, pp. 154-158, 1995. 


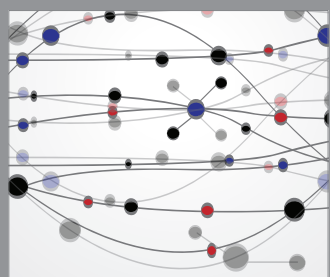

The Scientific World Journal
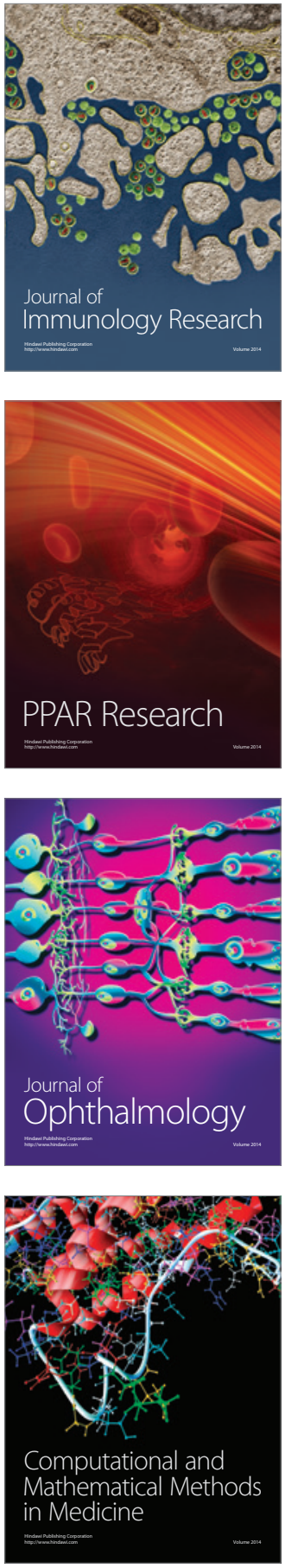

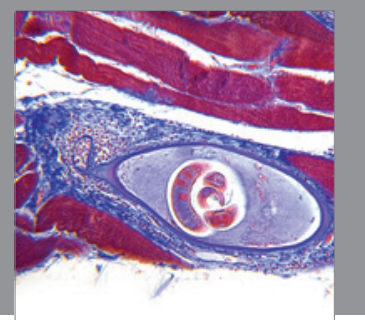

Gastroenterology

Research and Practice
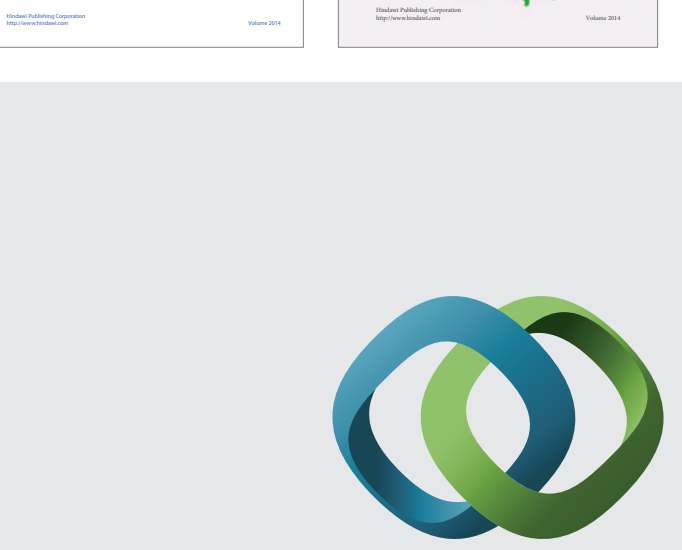

\section{Hindawi}

Submit your manuscripts at

http://www.hindawi.com
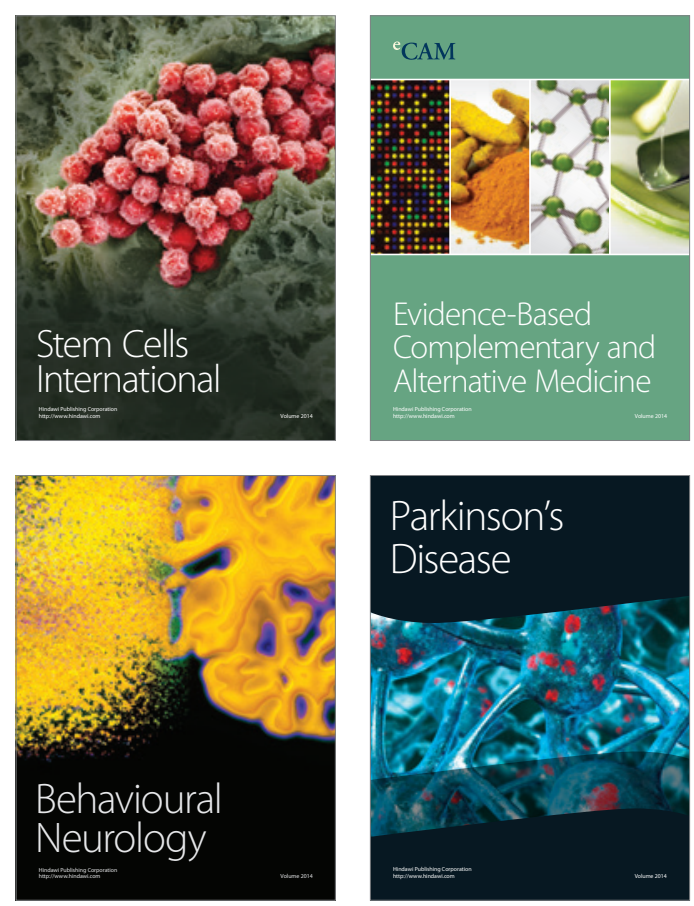

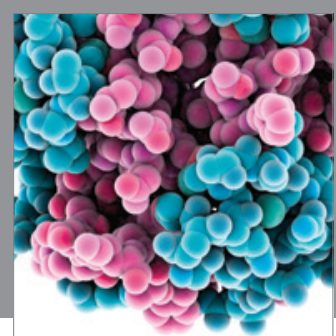

Journal of
Diabetes Research

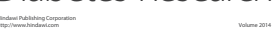

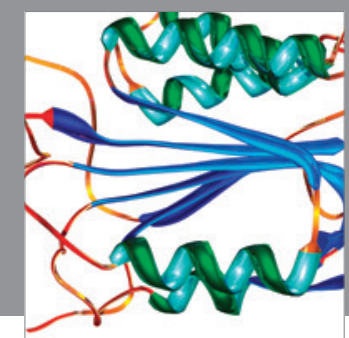

Disease Markers
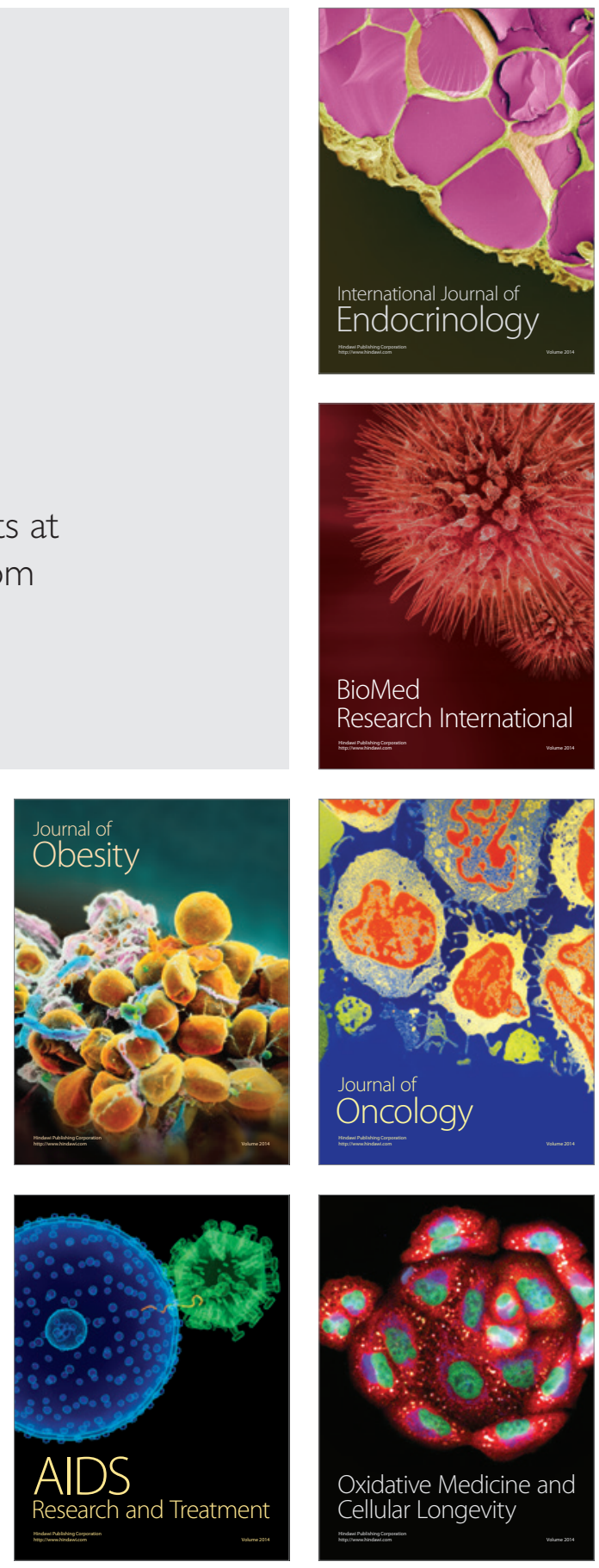